\title{
IMMUNIZATION COVERAGE, IN CHILDREN 12 YEARS OLD, IN WESTERN GREECE 4 YEARS EXPERIENCE
}

I. Giannakopoulos ${ }^{1}$, I. Christopoulou ${ }^{1}$, S. Fouzas ${ }^{1}$, G. Paraskevopoulos ${ }^{2}$, S. Tsironis ${ }^{3}$, I. Anastasopoulos ${ }^{2}$, G. Dimitriou 1

1 University Hospital of Patras, Paediatrics, Patras, Greece

${ }^{2}$ Administration of Primary Education, Achaia, Patras, Greece

${ }^{3}$ District General Hospital "St. Andrew", Physiotherapy, Patras, Greece

\section{Background}

Immunization coverage in children in Greece has achieved high levels during the previous years. However, recent poor economic situations in the country may have influenced children's admissions to pediatric surgeries, especially for preventive medicine.

The aim of this study was to investigate the immunization coverage in school-aged children of Patras (Western Greece).

\section{Methods:}

There were randomly selected 41 from 87 public primary schools in Patras, all in urban areas. All children who were born between 2003 and 2004, and whose parents gave consent, were included in the study. Immunization data were collected through the National pediatric vaccination cards. The sampling took place between September 2015 and December 2017.

\section{Results}

There were 1350 subjects enrolled in the study. The mean age was 12 years and $46.5 \%$ were boys. Children were fully vaccinated against Hepatitis B (98.9\%), Tetanus, Poliomyelitis, Diphtheria, Pertussis and Haemophilus Influenzae (98.3\%). Additionally, they were fully vaccinated against Measles, Mumps, Rubella (98.4\%), Varicella (82.4\%), Hepatitis A (84.7\%) and Meningitidococcus type C (97.1\%). The vaccination coverage was low for Streptococcus Pneumoniae (55.2\%), Mycobacterium (BCG, 24\%) and Meningitidococcus type B (3.6\%).

sex of the participants

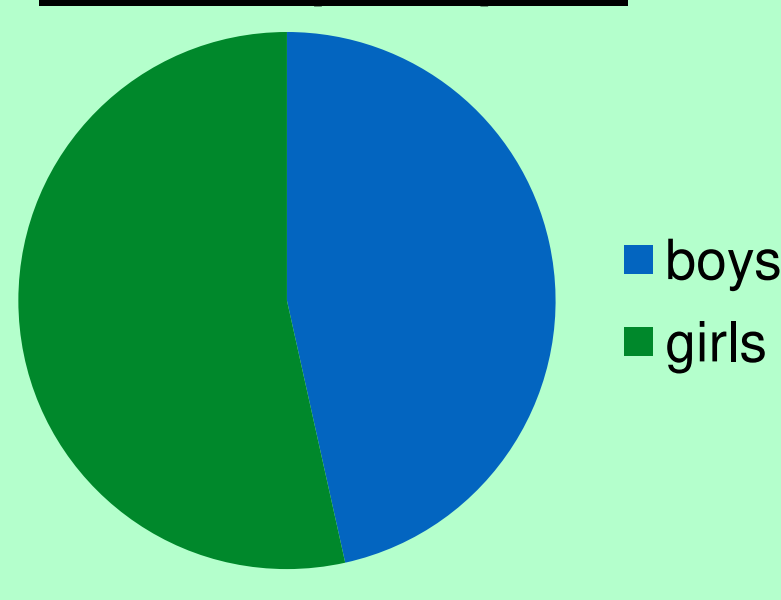

\section{Vaccine}

Measles,Mumps, Rubella

HBV

Poliomyelitis

Diphtheria

Pertussis

Haemophilus Influeanzae

Meningitidococcus type C

HAV

VZV

Streptococcus Pneumoniae

Mycobacterium

Meningitidococcus type B
Number of children vaccinated

1328

1335

1327

1327

1327

1327

1311

1143

1112

745

324

49
Vaccination coverage (\%)

98,4

98,9

98,3

98,3

98,3

98,3

97,1

84,7

82,4

55,2

24

3,6

\section{Conclusions:}

Immunization coverage in children (12 years old) in Patras remained in high levels. A lower vaccination coverage was observed against Streptococcus Pneumoniae. Greek children were almost not vaccinated against Mycobacterium and Meningitidococcus type B. The reasons of this low vaccination coverage should be explored. We should mention that the Meningitis B vaccine is not yet included in the National Vaccination Program. There is a need for a national strategy plan in order to increase vaccination, especially against Streptococcus Pneumoniae, Mycobacterium and Meningitidococcus (type B).

Presented in the 36th Annual Meeting of the European Society for Pediatric Infectious Diseases, MALMO,

SWEDEN, May 28- June 2, 2018

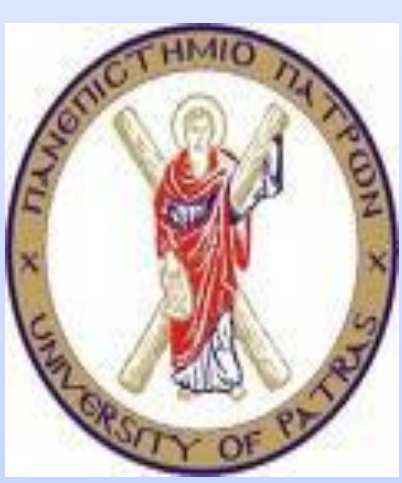

